\title{
Design and Application of Automatic Feeding Function for Ultrahigh Speed Cigarette Packing Equipment PROTOR-M8
}

\author{
Ming Chen, Yuming Liu, Wanli Liu, Jianxiong Qiu and Wei Wang \\ China Tobacco Zhejiang Industrial Co. LTD, Hangzhou, China
}

\begin{abstract}
In this paper, through the research on the automatic feeding function for ultrahigh speed cigarette machine PROTORM8, we build system architecture of automatic feeding mode, designing workflow and human-computer interaction interface, and planning AGV travel paths of BD pallet recovery and supplement. It has realized automatic supply of PROTOR-M8, which improves the production efficiency and automation level, and provides a reference for the similar system construction.
\end{abstract}

Keywords-automatic feeding function; tobacco industy logistics; PROTOR-M8; AGV; WMS

\section{INTRODUCTION}

Hangzhou Cigarette Factory introduced 5 sets of PROTORM8 after "11th Five-Year" technical innovation. The introductions of five sets of PROTOR-M8 are leading level of ultrahigh speed cigarette packing equipment in the world (production capacity of 20000 ton / min). [1]

The ROTOR-M8 which designed to have a separate automatic paper feeding system and a gripping device is a closed space formed by a safety door. Therefore, at the beginning of M8 putting into operation, the machine accessories pallet are realized by the "two supply" model, which resulted in the waste of labor, long feeding time and falling short of M8's automation degree.

Based on the above situation, Hangzhou cigarette factory first proposed and designed the automatic feeding function of M8,which provides a solution of connection of automated logistics system and cigarette equipment.

\section{DESIGN CONTENT}

\section{A. Automatic Feed Mode System Architecture}

AGV (Automated Guided Vehicle) acronym, which means "automatic guided vehicles", is equipped with a automatic guided electromagnetic or optical device, it can travel along the guide path specified, with safety protection and various transport vehicle shift function.[2]

WMS is a warehouse management system (Warehouse Management System) for warehouse management system that is through the storage business, outbound operations, warehouse allocation and other functions, effective controlling and tracking warehouse logistics and costing management of the whole process, which achieve and improve the enterprise warehouse information management.[3]

Ultrahigh speed cigarette packing equipment PROTOR-M8 automatic's feeding mode system architecture is shown in FIGURE I. The WMS and BD, the WMS and AGV, are connected through Ethernet. AGV and $\mathrm{BD}$ do not directly communicate. They exchange information through WMS. In order to ensure the safety of the AGV and BD, safety interlock information interface protocol is designed specifically. The safety interlock information is the required linkage information between the AGV and the $\mathrm{BD}$ when the $\mathrm{AGV}$ enters the $\mathrm{BD}$ safety door. And it is connected through TCP / IP, unified by the WMS.

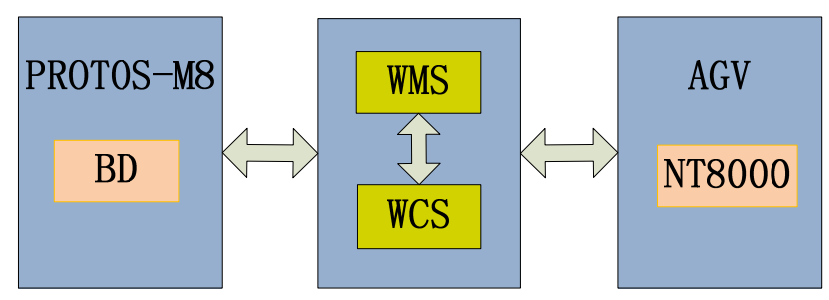

FIGURE I. PROTOR-M8 AUTOMATIC FEEDING MODE SYSTEM ARCHITECTURE

\section{B. Process of Automatic Feeding Mode}

When BD paper feeding device sensor detects the accessories on the pallet or tipping paper of cigarette paper number less than 1, BD sends "pallet replacement request" through the LAN communication system covering the location of the machine, the type of paper, the amount of paper. WMS quickly responds to the request and generates the automatic feeding task. [4]

From the security point of view, AGV sets the safety interlock signal and manual confirmation before entering the $\mathrm{BD}$. Through the verification, when $\mathrm{BD}$ opened the door to allow AGV to enter, the WMS sets the parameter of AGV, and the operator confirms the safety of AGV, pressing the "enter" button, which ensures the safety.

ROTOR-M8 BD automatic feeding process is shown in FIGURE II.

AGV information exchange in loading: <1>AGV travels to the BD security front position; <2> AGV send "AGV loading application"; $<3>$ the security door system open the security 
door after determining the robot position and the status of cargo loading status ; $<4>$ When the security door opens, the BD system sends the message "Allow AGV to enter the BD"; <5> $\mathrm{AGV}$ enters the platform to carry out the loading work; $<6>$ AGV comes out and sends the "Loading Complete" message after the job is finished; $<7>$ The door closes automatically.

AGV information exchange in unloading: $<1>A G V$ travels to the $\mathrm{BD}$ security front position; $<2>\mathrm{AGV}$ send "AGV unloading application"; $<3>$ t the security door system open the security door after determining the robot position and the status of cargo loading status $;<4>$ When the security door opens, the $\mathrm{BD}$ system sends the message "Allow AGV to enter the unloading"; $<5>$ AGV enters the platform to discharge the cargo; $<6>$ AGV sends out the "unloading complete" message after the job is finished; $<7>$ The door closes automatically.

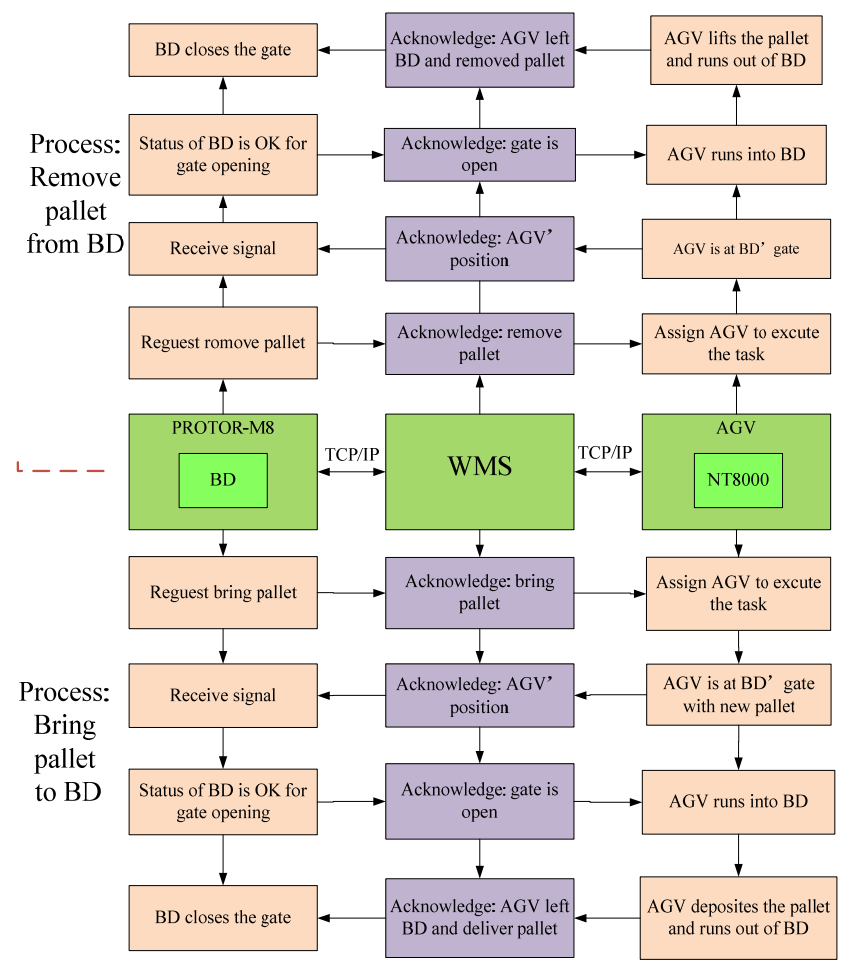

FIGURE II. PROCESS OF M8’ AUTOMATIC FEEDING MODE

\section{M8 Man-machine Interface Function Design}

In order to facilitate the operator to view and use BD automatic feeding, we design M8 man-machine interface function, as shown in FIGURE III. Under the operation panel menu, the trigger status of the function button is gray; we can immediately determine whether or not the automatic feeding function is open through the "start pallet supply" and "stop pallet supply" button status. The number of paper on pallet can also be viewed directly on the interface. When the automatic feed task is generated, the "pallet request" signal will be triggered and the button will be grayed out. "Reset interface" function can reset BD automatic call interface data. [5]

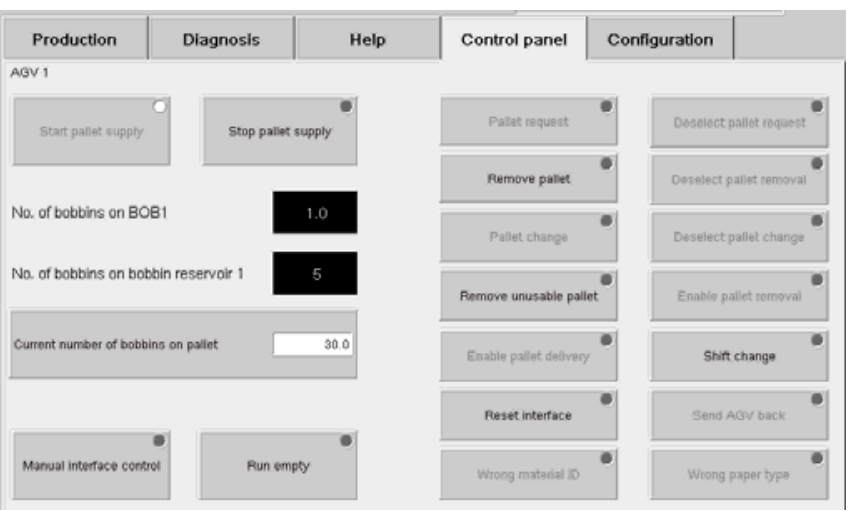

FIGURE III. AUTOMATIC FEED MAN-MACHINE INTERFACE OF BD

Considering the material error prevention, a level of automatic verification of accessories is built. In the BD operation interface, we set up the auxiliary material code, pallet type and quantity data. Specific settings are shown in FIGURE IV. Including the identification code mask of cigarette accessories of cigarette paper and tipping paper, namely the barcode recognition, and the correct identification code. In the process of data transmission between WMS and ROTOR-M8 BD, when the data is not consistent with the $\mathrm{BD}$, the door will not open automatically and display the wrong information on the BD interface.

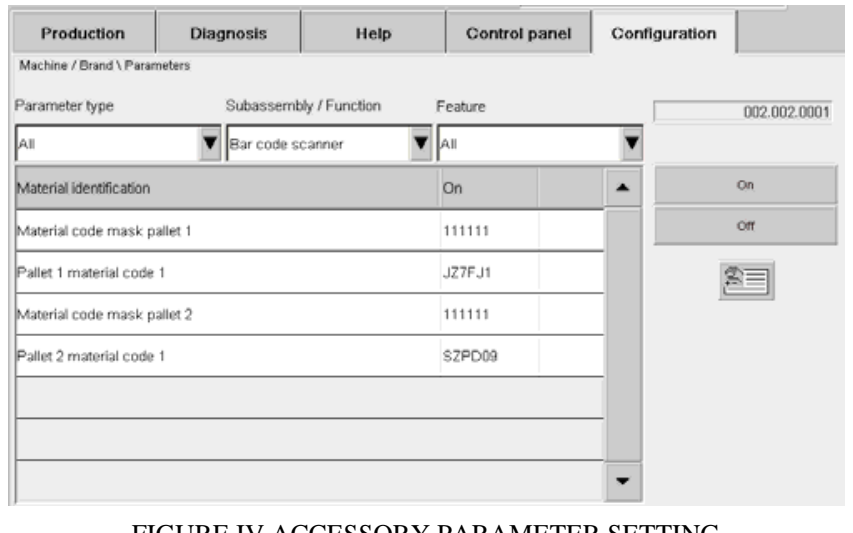

FIGURE IV ACCESSORY PARAMETER SETTING

We can also see the communication between the WMS and BD in the interactive panel, as shown in FIGURE V, WMS and BD communication instructions between the codes, such as "10" on behalf of the machine called material. BD automatically call the process by sending the code to inform WMS, WMS according to the corresponding request for material or empty tray recycling operations. 


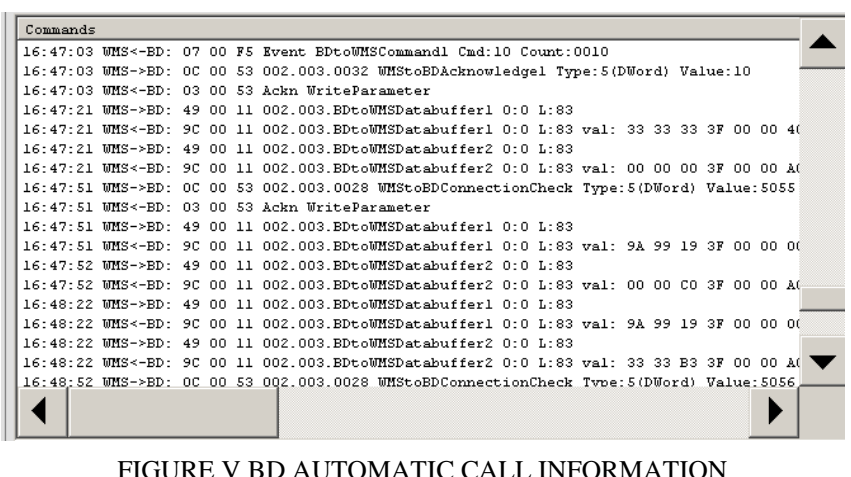

FIGURE V BD AUTOMATIC CALL INFORMATION

In addition, the BD interface also has a number of user interaction capabilities, so the operator can operation the process of BD feeding mode automatically, adjusting the flexible production needs.

\section{Pallet Storage Location and AGV Path Planning}

According to the best position of the manipulator in the BD work area to grab the accessories, we set the pallet location. At the same time, the pallet guide in the $\mathrm{BD}$ working area can determine the correctness of the position. If the pallet position is not correct, the pallet signal indicator light on the BD safety door will flash.

After confirming the location of pallet, in AGV scheduling system, we add the tobacco material automatic transmission of ROTOR-M8 (A1-A5), planning route AGV, and simulating experiments to verify the accuracy. [6]

Hangzhou Cigarette Factory AGV Conveyor System

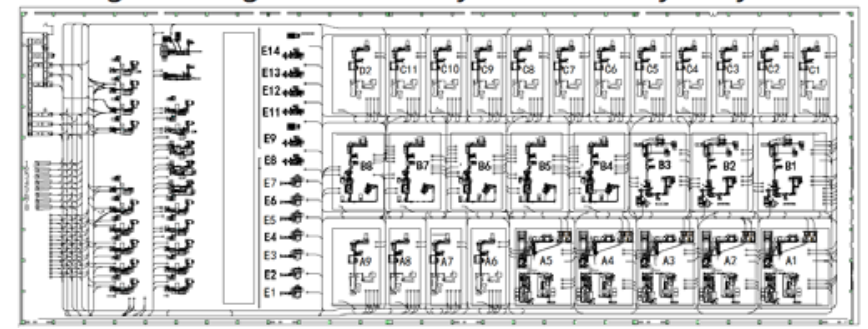

FIGURE VI. AGV TRAVEL PATH

\section{CONCLUSION}

Through the design and application of the BD automatic feeding function of M8, the "two feeding" mode is replaced. The data show that the average of feeding time of M8 is shortened from 40.6 to 23.9, saving $42 \%$ required time. Secondly, reducing the amount of labor, statistics show that the operator can save an average of 37.6 minutes every day. Through the provision of auxiliary materials in the BD code, we improve the ability of error proofing accessories. Finally, it realizes the automation and intelligent management of the workshop cigarette logistics transportation process, which provides a reference for the industry.

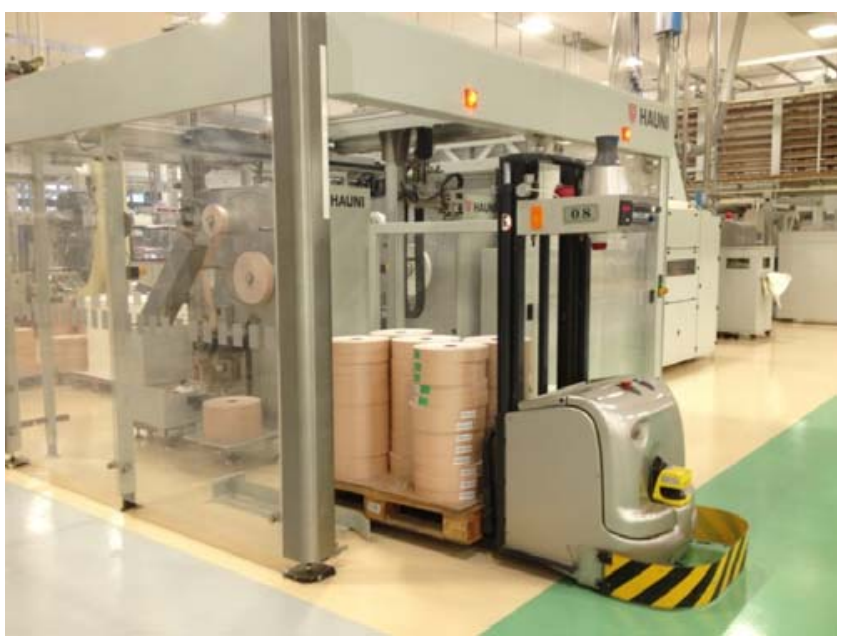

FIGURE VII. THE SCENE OF AUTOMATIC FEEDING INTO IMPLEMENT

\section{REFERENCES}

[1] Chen Ming, Ni Xiongjun, Zhang jun, Li Xiaona, Le Huan. Research and Application of AGV Technology in Tobacco Industry Logistics System [J]. Logistics technology and Application,2015(10):170-173.

[2] Sun Yu, Zhang Qian, Jiang Yiding. Application of AGV Controll System in Tobacco Logistics Process[J]. Computer application, 2012(05):39-41.

[3] Huang Dongsheng. Explore the use of AGV trolley in automated logistics system[J]. Science and Technology Innovation, 2013(36):55.

[4] Chen Ming. An automatic dispensing system for ultrahigh speed cigarette machine: China: CN201610216676.3[P].2016-09-07.

[5] Chen Ming. A method of recovering empty pallets for ultrahigh speed cigarette machine: China, CN201610219464.0[P].2016-07-20.

[6] Chen Ming. A method of feeding material pallet for ultrahigh speed cigarette machine: China, CN201610221107.8[P].2016-07-27. 